دراسة بعض معايير الام في اناث الفئران البيض المجرعة بالمستخلص المائي الخام لبذور فول الصويا (Glycine max)

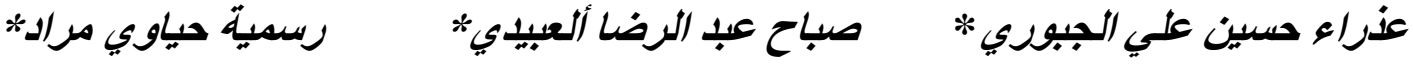 \\ استلام البحث 3، حزير ان، 2010 اناول، 2010 \\ قبول النشر 26، تشرين الاول، حزير 2010
}

تمت دراسة التأثير المحتمل للمستخلص المائي الخام لبذور فول الصويا على بعض المعايير الدموية

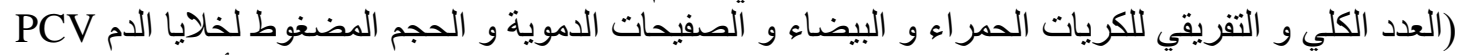

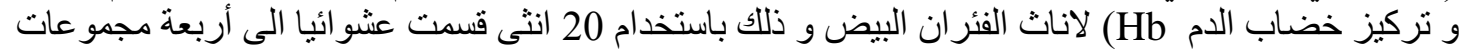

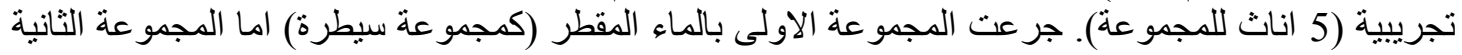

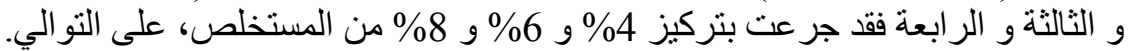

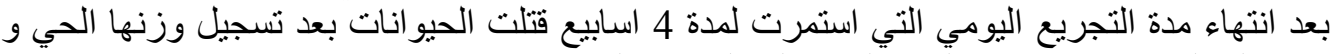

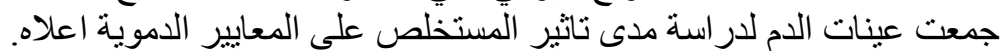

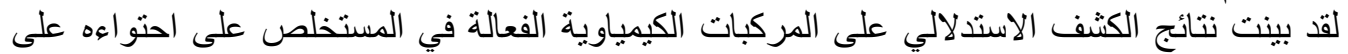

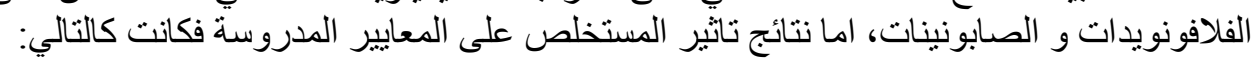

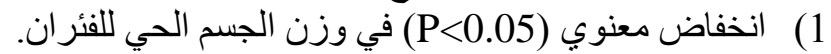

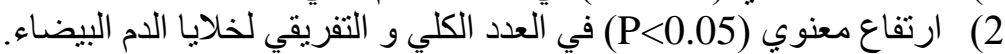

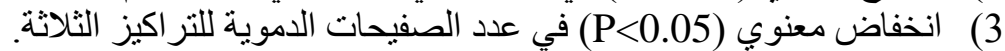
4) انخفاض معنوي (P<0.05) و (P<0.01) في (P<01) في حجم خلايا الدم المضغوط و العدد الكلي لكريات الدم

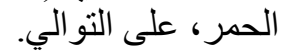

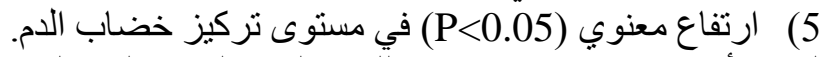

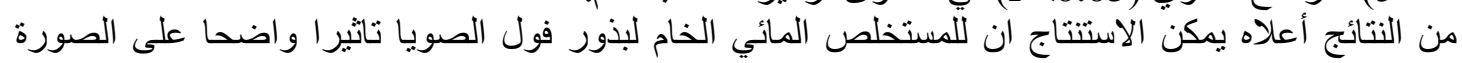

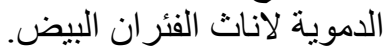
الكلمات المفتاحية: المستخلص اليفل المائي لبذور فول الصويا، عوامل الام، اناث الفئران.

الأهمية الطبية لبذور فول الصويا متأتية

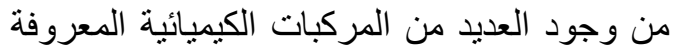

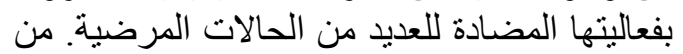

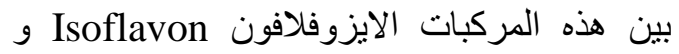

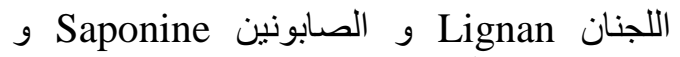

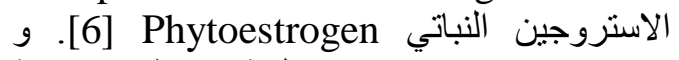
يعتبر الاخير مهماً في معالجة اضطر اب نسبة الاستروجين الطبيعي في الجسم.

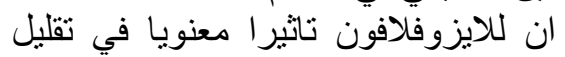
الإصابة بأمراض القلب التاجية عن طريق تقليل مستوى الكولسترول [7] اضنافة الى إدامته للأو عية الأية

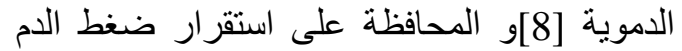
الثرياني [9] من خلال احتواء بروتينات الصويا على الجنسنين و الديادزين.

كما وجد[10]ان فول الصويا يقل يقل من

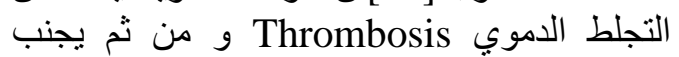
الإنسان مشكلة التعرض للازيل الإمات القلبية و السكتات

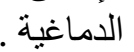

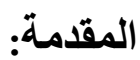

يعود فول الصوفة Glycine max الى الى العائلة

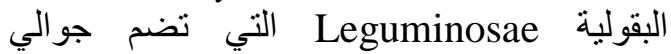

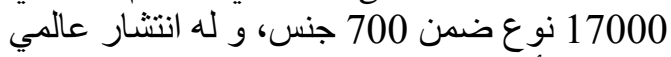

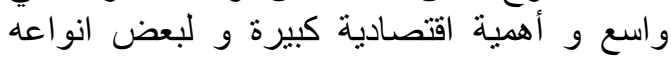

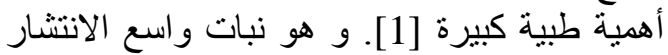

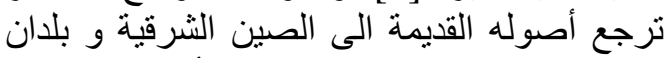

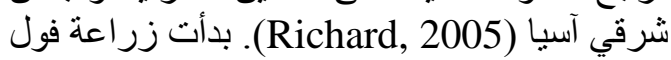

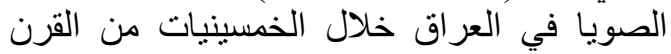
الماضي، لكن ماز الت زراف خلاف الته محدودة في نطاق من الترن

يمنالك فول الصويا أهمية غذائية كونه

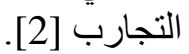

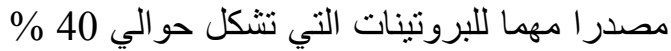

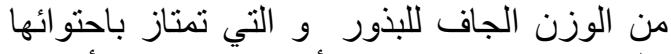
على الاحماض الامينية الأساسية و غير الأنيا لأساسية [4,3]. و تحتوي البذور ايضا على الدهون الإنية الخالية

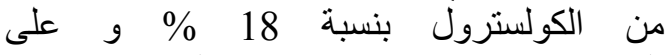
الكربو هيدرات بنسبة 30 \% و و حو الي 14 \% \% منها

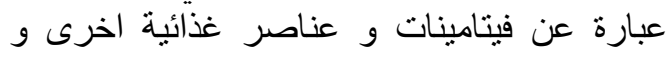
رطوبة و رماد [5]ة فينات

* قسم علوم الحياة، كلية العلوم للبنات، جامعة بغداد 
Rotary evaporator جهاز المبخر الدوار للحصول على المستخلص الجاف.

نم الكثف عن المركبات الكبميائية الفعالة

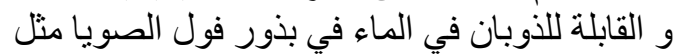

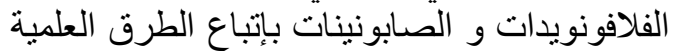
المتبعة في هذا المجال [13,12] على التوالي.

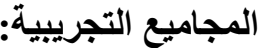

قسمت الفئران أنفة الذكر عشوائيا الى أربعة مجاميع (5 فئر ان للمجمو عة) و عوملت على النح النحو النيا الأتي:

مجموعة السيطرة (G-1) جرعت بـ 0.1 مل من الماء المقطر.

المجمو عة الثانية (G-2) جر عت بـ 0.1 مل بتركيز 4 \% من المستخلص.

المجموعة الثالثة (G-3) من الثرة جرعت بـ 0.1 مل بتركيز 6 \% من المستخلص.

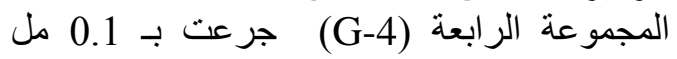
بتركيز 8 \% من المستخلص.

تم حساب تركيز الجرع باستعمال المعادلة

التالية: C Cavaging اسابيع فتمت عن طريق استعمال محقنه طبية نبيذة التردية

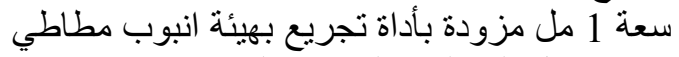

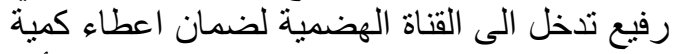

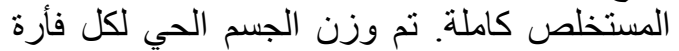

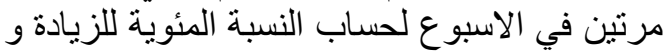

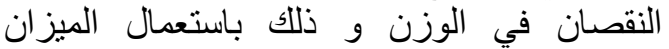
الالكتروني الحساس. بعد الانتهاء من مدة التجريع التهان تم ايضا تسجيل وزن الجسم الحي للحيوانات بعدهات التهات

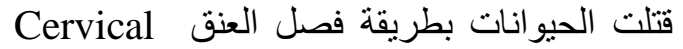
dislocation

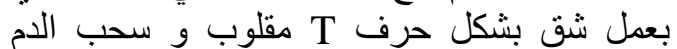
بواسطة طعنة القلب باستعمال المحقنة الطبية.

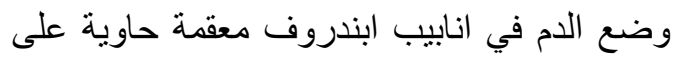

بعد الانتهاء من جمع نماذج الدم تم اجر اء اء الثري

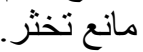
العد الكلي لخلايا الدم البيضاء و و كريات الداء الداء الداء

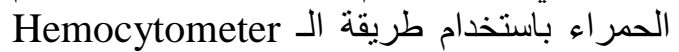

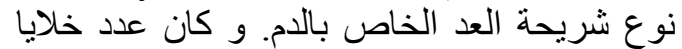

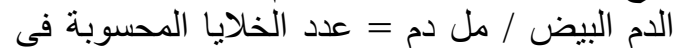

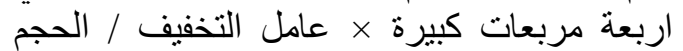

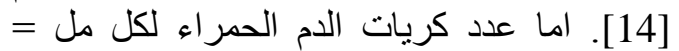
عدد الكريات المحسوبة × 14.01 × 0.00000

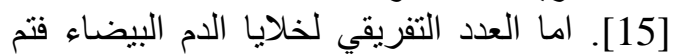

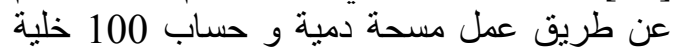
بيضاء بكافة أنواعها و من ثم استخرجت دمث النسبة

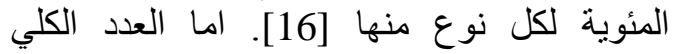
للصفيحات الدموية Platelats Count فتم حسابه هو الآخر باستخدام طريقة العد الخاصة بخلايا الدم
بناءا على ما هو معروف من تأثيرات

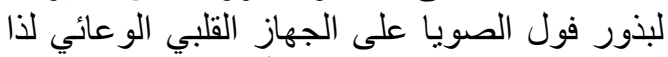

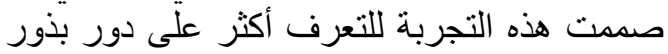

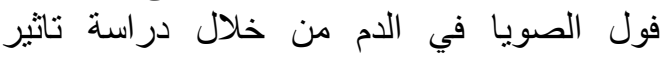
مستخلصها المائي الحار الخام على بعض الثان المعايير الدموية في اناث الفئران البيض.

\section{المواد و طرائق العمل: المادة النباتية:}

Plant Material تم الحصول على المبادة المبادة

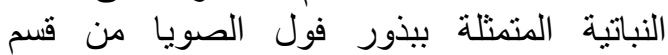

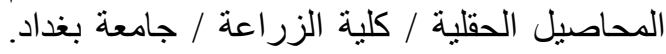

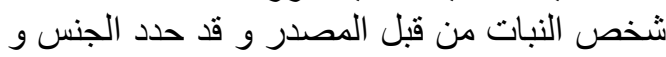
النوع بما يلي: Glycine max (Le).

حيوانات التجربة: Experimental Animals استخدم في هذه الدراسة 20 انثى بالغة من

الفئران البيض Albino Female Mice نوع فئن سلالة Balb/c musculus

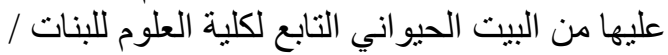

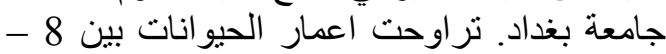

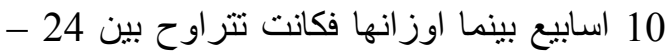
26 غم عند بداية التجربة.

وضعت الحيوانات في أقفاص بلاستيكية

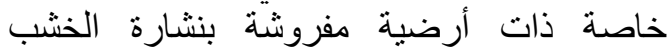
الناعمة. تم إيواء الحيوانات في البيت الحئة الحيواني

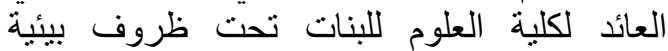
متشابهة بدرجة حرارة ثابتة بحدود 25 مج و و نهوية

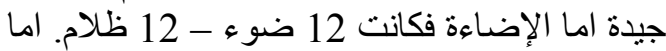

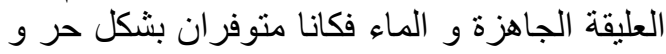
مستمر ad libitum و بكميات كافية طيلة مدة الجناء

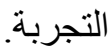

لتحضير المستخلص المائي الحار الخام

تحضير المستخلص: لمسحوق بذور فول الصويا اتبعت طريقة [11].

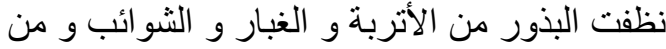
ثم طحنت بالمطحنة الكهربائية. ثم اخذ 100 غم من الكن

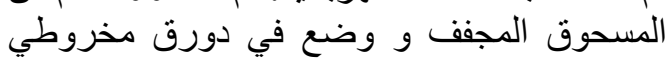
سعة 1000 مل. ثم اضيف اليه 500 مل من المباء

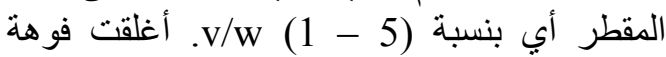
الدورق بالقطن الطبي بعدها وضيع المزيج في حمام

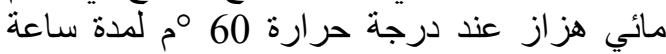

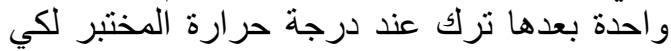
يبرد. بعد مرور 24 ساعة على على هذه الحالة الحة رشح

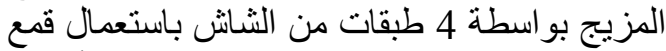
بخنر مع ورق الترشيح. وزع الر اشح على على أنابيب

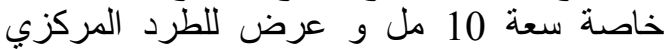
المبرد بسر عة 2000 دورة/دقيقة لمدة 10 دقائق. للردي. أهمل الراسب و اخذ الر اشح و ركز بوضعة فئ في 
جذول ( 1 ) ) : المعدل ( الخطأ القياسي للنسب المئوية للزيادة و النقصان في وزن الجسم الحي (غرام) للفئران

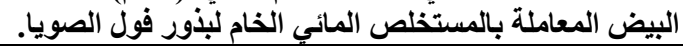

\begin{tabular}{|c|c|c|c|c|c|}
\hline \multicolumn{4}{|c|}{ النسبة المئوية للزيادة و النقصسان } & \multirow{2}{*}{ التجريع بَلِ } & \multirow{2}{*}{ 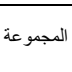 } \\
\hline الاسبوع 4 & الاسبوع 3 & الاسبوع 2 & الاسبوع 1 & & \\
\hline \pm 108.63 & \pm 103.11 & \pm 105.60 & \pm 103.63 & & \\
\hline 1.61 & 1.16 & 1.79 & 1.44 & 26.23 & السيطرة \\
\hline \pm 75.74 & \pm 76.58 & \pm 82.71 & \pm 91.94 & 24,2 & 04 \\
\hline$\cdot 1.41$ & $\cdot 1.26$ & $• 1.81$ & $\cdot 1.39$ & 24.23 & $\% 4$ \\
\hline \pm 91.04 & \pm 91.68 & \pm 92.65 & \pm 95.13 & 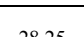 & 0 \\
\hline$\cdot 1.98$ & $\bullet 1.45$ & $\cdot 2.20$ & $\cdot 2.36$ & 28.25 & $\% 0$ \\
\hline \pm 65.65 & \pm 68.55 & \pm 70.40 & \pm 80.18 & & \\
\hline$\cdot 1.67$ & $\cdot 1.13$ & $\cdot 2.30$ & $\bullet 1.84$ & 23.12 & $\% 8$ \\
\hline
\end{tabular}

* - الفروق معنوية ( 0.05 > P ) بالمقارنة مع السيطرة.

\section{اظهرت النتائج المذكورة في جدول رقم 2}

ان العدد الكلي و التفريقي لخلايا الدم اليضيض اليض ازداد

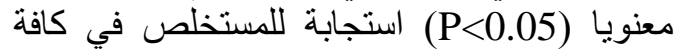

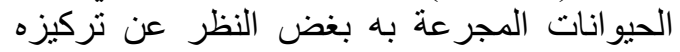

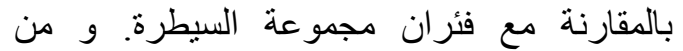
الملاحظ ان هذه الزيادة جاءت طرئ طردية مع زيادة

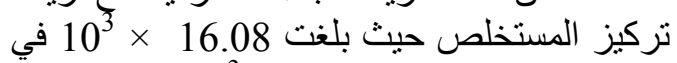

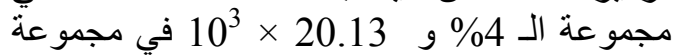

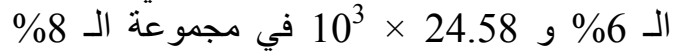
مقارنة بمجمو عة السيطرة التي كان العدد الكلي فيها 10 6.71

يعتقد ان السبب الرئيسي في هذا الارتفاع

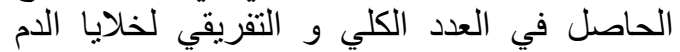

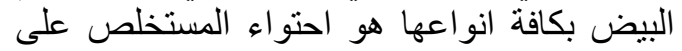
مادة الصابونين ذات الآثار المشخصة الثراه على الجهاز

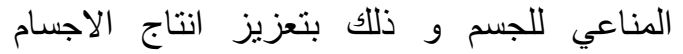

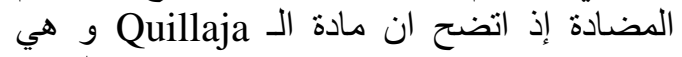
مركب صابونيني تزيد من سرعة النتاج النيح الخلايا

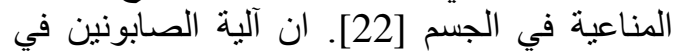

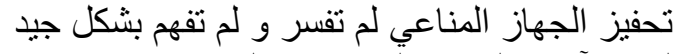
لحد الآن و لو ان الكثير من التفسيرات قدمت لفئ و

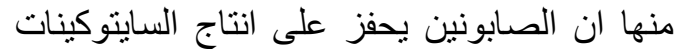
(بروتينات استجابية) و الانترلوكينات (بروتينات الاتينات

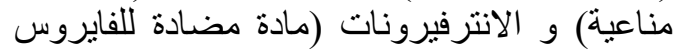

في الدم) و التي تتوسط التحفيز المناعي [24,23].

جدول ( 2 ) : المعدل \pm الخطأ القياسي للعدد الكلي (ملم ج (1030) و التفريقي ( \% ( ) ) لخلايا الدم البيض في الفئران المجرعة بالمستخلص المائي الحار الخام لبذّر فول لخران

\begin{tabular}{|c|c|c|c|c|c|c|}
\hline B & E & M & L & N & W.B.C & الصجوع \\
\hline \pm 0.16 & \pm 0.13 & \pm 0.32 & \pm 3.22 & \pm 2.87 & \pm 6.71 & \\
0.02 & 0.02 & 0.07 & 0.17 & 0.17 & 0.28 & \\
\hline \pm 0.36 & \pm 0.26 & \pm 0.97 & \pm 11.13 & \pm 3.34 & \pm 16.08 & $\% 4$ \\
$* 0.02$ & $* 0.02$ & $* 0.06$ & $* 0.10$ & $* 0.04$ & $* 0.06$ & \\
\hline \pm 0.42 & \pm 0.38 & \pm 1.21 & \pm 13.00 & \pm 5.12 & \pm 20.13 & $\% 6$ \\
$* 0.02$ & $* 0.01$ & $* 0.04$ & $* 0.13$ & $* 0.14$ & $* 0.09$ & $\%$ \\
\hline \pm 0.48 & \pm 0.46 & \pm 1.38 & \pm 16.20 & \pm 6.06 & \pm 24.58 & $\% 8$ \\
$* 0.03$ & $* 0.02$ & $* 0.07$ & $* 0.09$ & $* 0.13$ & $* 0.38$ & \\
\hline 0.07 & 0.11 & 0.18 & 1.09 & 0.32 & 7.29 & LSD \\
\hline
\end{tabular}

و بتطبيق المعادلة التالية : عدد الصفيحات الدموية

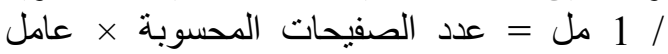
التخفيف × عدد المربعات المتوسطة [17].

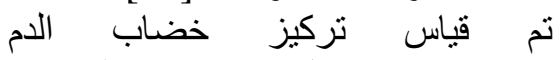
بemoglobin الضوئي Spectrophotometer بطول موجي مقداره 540 نانوميتر. و بتطبيق المعادلة التالية حصلنا على قيمة التركيز :

$\mathrm{Hbg} / \mathrm{dl}=(\mathrm{A} 540$ of test sample $) /$ (A540 of standard) $\times$ (concentration of standard $) \times($ dilution factor/1000)

حجم الكريات المضغوطة Packed cell volume (PCV)

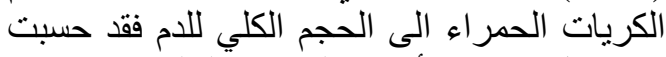

باستعمال طريقة الأنبوب الثعري الثقليدية [15].

التحليل الإحصائي: عرضت النتائج التي حصلنا عليها من التجربة على التئ

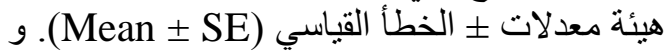
لتحديد الفروقات المعنوية بين المتوسطات استخدم اختبار انوفا (ANOVA) عند مستوى احتمالية

.[18] $\mathrm{P}<0.05$

النتائج و المناقشة:

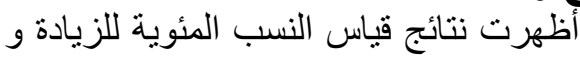

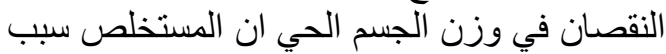
نقصان معنويا (P<0.05) في وزن الجن الجسم اللفئران

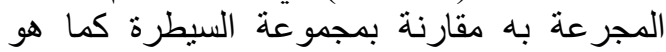

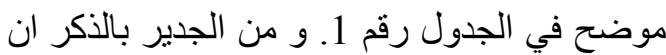

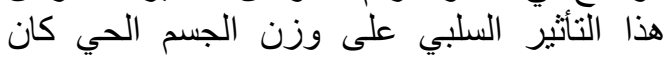

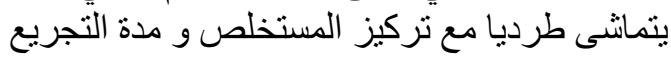

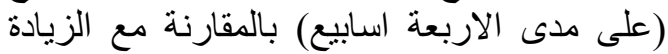

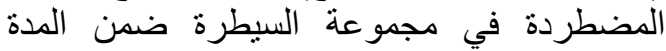
نفسها، حيث كانت النسبة المئوية للزيادة في في في النية مجموعة السيطرة هي 8.36\% من وزن الجسم للجي الحي قبل بدأ تجريع المستخلص الهي بينما كان

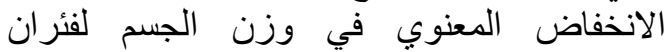
المجاميع التجريبية الثنلاثة الاخرى (4) و

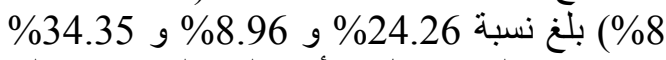

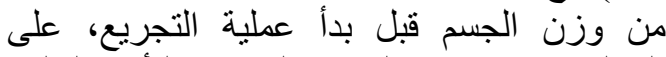
التوالي. توضح هذه الفروق المعنوية التأثير السلبي التي لئي

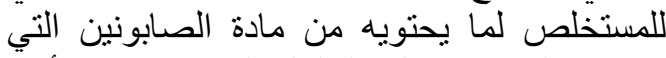
يعتقد ان لها تاثير على السلوك الغذائي. هذا ما أكده

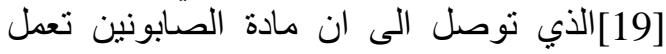

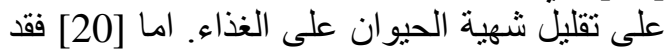

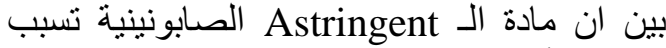

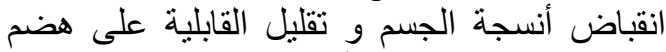

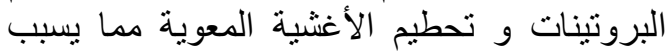
تثبيط عملية نقل الغذاء داخل الجسم [21]. 
PCV ما توصل البه[30] عند دراسته لتأثير الصابونين على نشاط الأغشية الخلوية.

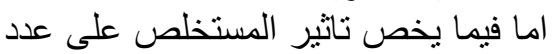

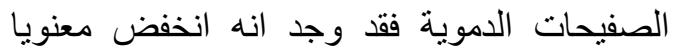

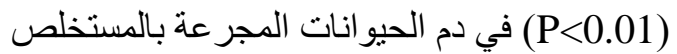
و كان هذا الانخفاض كبيرا كلما ازداد تركيز بالديز المستخلص (جدول 3).

جاءت هذه النتائج متو افقة مع ما توصل التول

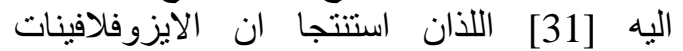

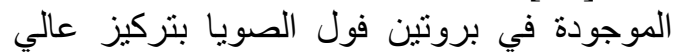

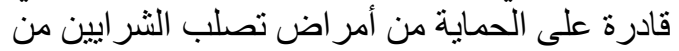
خلال تثثيطها لنشاط الصفيحات النيحات الدموية و تجمعها

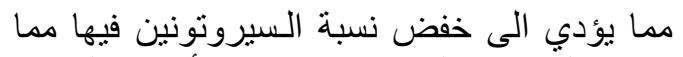
يؤدي الى منع التخثر و انسداد الأوعية الدموية التية

جدول ( 3 ) : المعدل ـ الخطأ القياسي لعدد كريات الدم

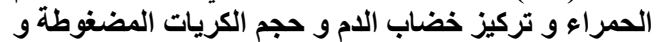

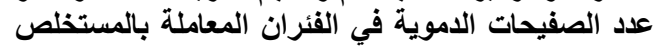

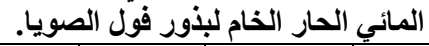

\begin{tabular}{|c|c|c|c|c|}
\hline عدلا الصففيحات & 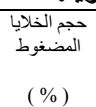 & خضاب الدم & 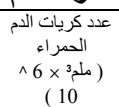 & المجاميع \\
\hline $\begin{array}{c} \pm 175.285 \\
2.36\end{array}$ & $\begin{array}{c} \pm 38.892 \\
0.82\end{array}$ & $\begin{array}{c} \pm 8.99 \\
0.26\end{array}$ & $1.18 \pm 85.16$ & السيطرة \\
\hline $\begin{aligned} \pm & 153.142 \\
& \cdot 1.91\end{aligned}$ & $\begin{array}{c}37.811 \\
0.69\end{array}$ & $\begin{array}{l} \pm 11.73 \\
0.44\end{array}$ & $\begin{array}{c}1.03 \pm 63.44 \\
\cdot\end{array}$ & $\% 4$ \\
\hline $\begin{array}{c} \pm 138.428 \\
\cdot 1.97\end{array}$ & $\begin{array}{c} \pm 32.542 \\
\cdot 0.66\end{array}$ & $\begin{array}{l} \pm 12.08 \\
0.40\end{array}$ & $\begin{array}{c}1.07 \pm 55.32 \\
\cdot\end{array}$ & $\% 6$ \\
\hline $\begin{array}{l} \pm 123.142 \\
\cdot 2.18\end{array}$ & $\begin{array}{c} \pm 28.621 \\
\cdot 0.29\end{array}$ & $\begin{array}{l} \pm 13.80 \\
\cdot 0.43\end{array}$ & $\begin{array}{c}1.30 \pm 39.08 \\
\cdot\end{array}$ & $\% 8$ \\
\hline 0.01 & 0.05 & 0.05 & 0.01 & LSD \\
\hline
\end{tabular}

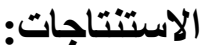

مما تقدم من معطيات لهذه الدر اسة يمكننا القول

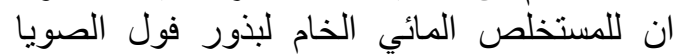

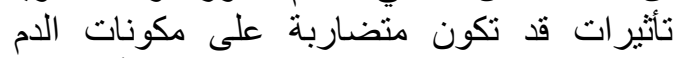

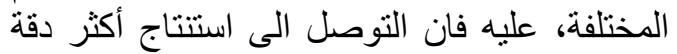
بحاجة الى در اسات أكثر عمقا في هذا المجال.

المصادر: - المي

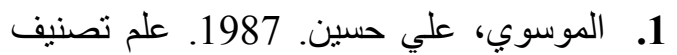
النبات، الطبعة الاولى، جامعة بغداد، صل صلص

.226

2. معيوف، محمود احمد. 1982.

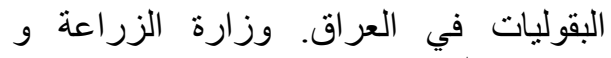
الاصلاح الزراعي. ص صل 285.

3. Schaafsma G 2000. The protein digestibility-corrected amino acid

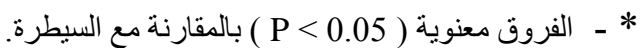

WBC = white blood cells, $\mathrm{N}=$ neutrophils, $\mathrm{L}=$ lymphocyte,

M = monocyte, $\mathrm{E}=$ eosinophil, $\mathrm{B}=$ basophil يتضمن الجدول رقم 3 معدل عدد كريات

الدم الحمر RBC و مستوى تركيز خضاب الدم و Hb و PCV الفئران البيض المجر عة بالمستخلص المائي الخام

$$
\text { لبذور فول الصويا. }
$$

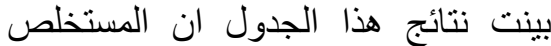

سبب انخفاضا معنويا عاليا (P>0101) في معدلات عدد الـ RBC و بصورة طردية مع زيادة تركيز

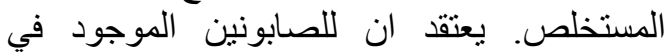

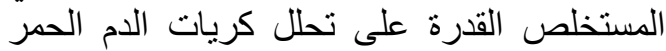
[25]. لقد استتتج [26] ان هذه الخاصية للصنابونين هي نتيجة ميله للاتحاد مع الجزء السكاء السكري لدهون

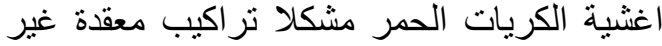

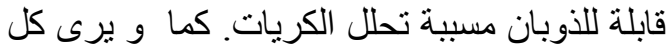

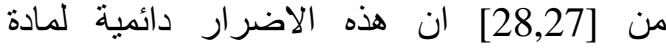
الصابونين و الكولسترول في اغثية الكريات

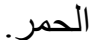

اما تركيز خضاب الدم فلم يزداد معنويا

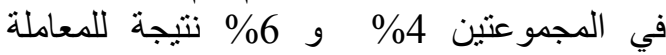

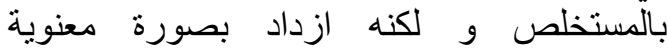

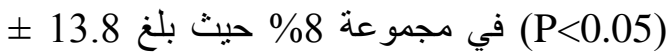

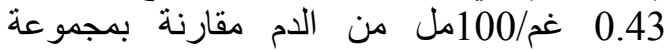

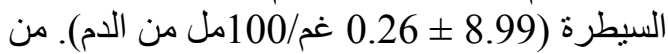
الجدير بالذكر لم يكن هناك أي تاتثير معنوي في

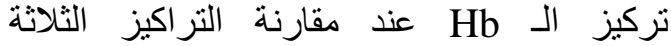
للمستخلص مع بعضهها.

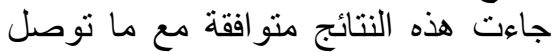
اليه [29] في در استه لتاثير فول الصويا في تركيز

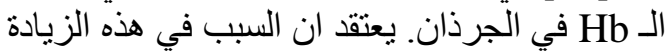

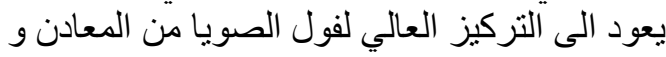

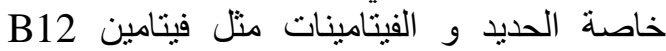

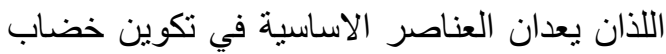

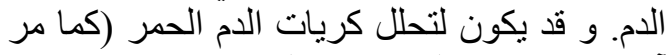
آنفا") و انسياب الـ Hb في البلازما سببا في ازدياد

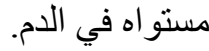
ان النسبة المئوية لحجم الكريات الحمر المضغوط PCV او الـ Hematocrit هي الاخرى قد تأثرت بالمستخلص حيث انخفضت غير معنو معنا

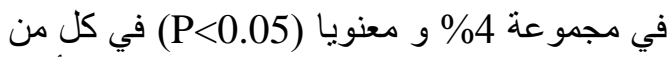

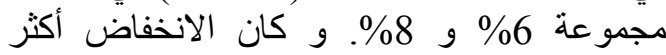

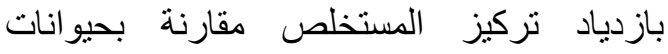

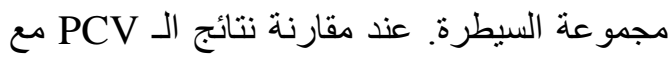

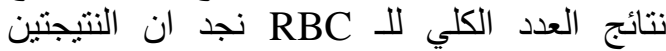

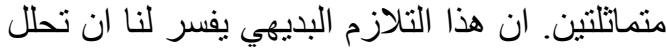

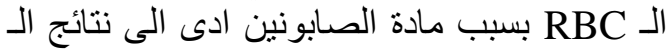


chines medicines. Shoya Kuhak Zasshi, 44: 225-229.

12. Jaffer, H.T.; Mahmoud, M.; Jawad, A.; Nagi, A. and Al-naib, A. 1983. Phytochemical and biological screening of some Iraqi plant. Fitoterapialix, pp. 299.

13. Harborne,J.B.1973. Phytochemical methods. Science paper backs, Chapman and Hall. London, UK.

14. Harris-Young, L. 1995. Principles of Hematology. Wmc. Brown Publishers, UK.

15. Wilkinson PC, Carmichael DS 1964. Journal of Laboratory Clinical Medicine 64, 529-539.

16. Myers, R.L. 1995. Laboratory manual of immunology. $2^{\text {nd }}$ ed. Wm.c. Brown Publishers. USA.

17. فرهاد، اكرم داود و قتبر، سروري علي. التئي

1986. التقنية الطبية. وزارة التعلية التعليم العالي و

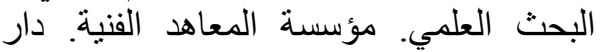

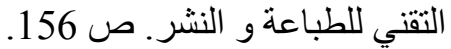

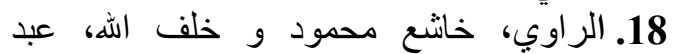

العزيز محمد 1980. 1لمع تصميم و و تحليل

التجارب الزربز اعية. وزارة التعليم العالي و و

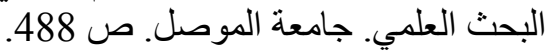

19. Oleszek W, Nowacka J, Gee JM, Wortley G, Johnson IT. 1994. Effects of some purified alfalfa (Medicago sativa) saponins on transmural potential difference in mammalian small intestine. Journal of the science of food and agriculture. 65, 35-39.

20. Francis, Zohar, Harinder P.S. and Klaus 2002. The biological action of saponins in animal system: a review. British Journal of Nutrition, 88(6): 587-605.

21. Shimoyamada M, Suzuki M, Sonta H, Maruyama M, Okubo K. 1998. Antifungal activity of saponin fraction obtained from Asparagus Officinalis L. and its active principle. Agriculture and biological chemistry 54, 25232557.

22. Oda K, Matsuda H, Murakami T, Katayama S, Ohgitani T, score. Journal of Nutritition 130, 1865-1867.

4. Cordle CT, 2004. Soy protein allergy : incidence and relative severity. Journal of Nutrition 134(5) : 1213-1219.

5. Messina, M.J. 1999. Legumes and soybeans: overview of their nutritional profiles and health effects. Am. J. Clin. Nutr. 70: 439450.

6. Venter, C.S. 1999. Health benefits of soybeans and soy products : a review. J. Fam. Ecol. Sci. 27: 2433.

7. Wiseman, H. 2000. Isoflavon phytoestrogens consumed in soy decrease $\mathrm{F}(2)$ isoproatane concentration and increase resistance of low-density lipoprotein to oxidation in humans. Am. J. Clin. Nutr., 72(2): 395-400.

8. Van der Schouw, Y.T. 2002. Higher usual dietary intake of phytoestrogens is associated with lower aortic stiffness in postmenpausal women. Arterioscler. Thromb. Vasc. Biol. 22(8): 1316-1322.

9. Teede, H.J.; Dalais, F.S.; Kotsopulos, D. 2001. Dietary soy has both beneficial and potentially adverse cardiovascular effects: a place-controlled study in men and postmenopausal women. J. Clin. Endoctinal. Metab., 86(7): 353-60.

10. Sacks FM, Lichtenstien A, Van Horn L 2006. Soy protein, isoflavones, and cardiovascular health : an American Heart Association Science Advisory for professionals from the Nutrition Committee. Circulation 113(7): 1034-44.

11. Zhang-mu. M. X. Sakai, Osei, T. Safo, H. Nasasoi, Hikito, M. Safo, M. Mizuno, K. Ono, H, Nakeneg 1990. Autimutogenic activity by the medical plant in traditional 
29. Seeman P 1974. Ultrastructure of membrane lesions in immune lysis, osmotic lysis and drug-induced lysis. Federation proceedings 33, 2116-2124.

30. Alada, A.R.A., Akande O.O, Ajayt F.F 2004. Effect of soybean diet preparations on some hematological and biochemical indices in the rate. African Journal of Biomedical Research, Vol.: 7, 71-74.

31. Melzig MF, Bader $G$ and Loose R. 2001. Investigation of the mechanism of membrane activity of selected triterpenoid saponins. Planta Medica. 67, 43-48.

32. Schoene NW and Guidry CA 1999. Dietary soya isoflavones inhibit activation of rat platelets. J Nutr Biochem; 10:421-426.

33. Williams JK and Clakson TB 1998. Dietary soy isoflavones inhibit in-vivo constrictor responses of coronary arteries to collagen-induced platelet activation. Coron Artery Dis; 9(11): 759-764.
23. Yoshikawa M. 2000. Adjuvant and haemolytic activities of 47 saponins derived from medical and food plants. Biological Chemistry 381, 67-74.

24. Jie YH, Cammisuli S, Baggiolini M. 1984. Immunomodulatory effects of Panax ginseung C.A. MEYER in the mouse. Agents and actions. 15, 386-391.

25. Kensil CR. 1996. Saponins as vaccine adjuvants. Critical reviews in the therapeutic drug carrier systems. 13, 1-55.

26. Hughes-Jones N.C. 1984. Saponins which are fat-solvents, may act on the red cell membrane disrupting the lipid components. Lecture notes on hematology, fourth edition, P: 172.

27. Goglein H and Huby A 1984. Interaction of saponin and diagitonin with black lipid membranes and lipid monolayers. Biochemica et Biophysica Acta. 773, 32-38.

28. Bangham AD and Horne RW. 1962. Action of saponins on biological cell membrane. Nature 196, 952-953. 


\title{
Some blood parameters study in albino female mice orally given crude aqueous soy bean (Glycine max) seeds extract
}

\author{
Athraa H. A. Al-Jiboory* Sabah A. R. Al-Obaidi* \\ Rasmiya H. Murad*
}

*College of Science for women/ University of Baghdad.

\begin{abstract}
:
The possible effect of the crude aqueous extract of soy bean seeds on some blood parameters (total count of red blood cells, white blood cell, (total and differential) blood platelates, packed cell volume and concentration of blood hemoglobin) was studied in 20 albino female mice which were allocated in four experimental groups ( 5 mice/group). The first group was orally treated with distilled water (control group) while the second, third and fourth group were given a concentration of $4 \%, 6 \%$ and $8 \%$ of the extract, respectively.

At the end of the daily gavaging, which lasted for 4 weeks, the animals were killed, after recording their life body weight, and blood samples were collected from each mice to study the effect of the extract on the above mentioned parameters.

Some of the active ingredients in the soy bean seeds extract were analytically tested. This test showed that the extract contained flavonoids and saponins.

The effect of the extract on the studied blood parameters is reflected by the following results:

1) Significant $(\mathrm{P}<0.05)$ decline in life body weight of the treated animals.

2) Significant $(\mathrm{P}<0.05)$ increase in total and differential count of the white blood cells.

3) Significant $(\mathrm{P}<0.05)$ decrease in number of blood platelets in all treated groups.

4) Significant $(\mathrm{P}<0.05)$ and $(\mathrm{P}<0.01)$ decrease in $\mathrm{PCV}$ and total count of red blood cells, respectively.

5) Significant $(\mathrm{P}<0.05)$ increase in blood $\mathrm{Hb}$ concentration.

From the above results it could be concluded that the crude aqueous extract of soy bean seeds has a direct negative effects on the studied blood parameters in albino female mice.
\end{abstract}

